\title{
Perfil hematológico de recém-nascidos de uma Unidade de Terapia Intensiva neonatal de Teresina - PI
}

\author{
Hematologic profile of newborns in a Neonatal Intensive Care Unit in Teresina - PI \\ Perfil hematológico de recién nacidos de una Unidad de Terapia Intensiva neonatal de \\ Teresina - PI
}

Natália Elias Ribeiro de Sá ${ }^{1 *}$, Roseane Mara Cardoso Lima Verde ${ }^{2}$, Matheus Hipólito do Nascimento ${ }^{3}$, Leonardo Ferreira Soares ${ }^{4}$, Evaldo Hipólito de Oliveira ${ }^{1,5}$.

\section{RESUMO}

Objetivo: Avaliar o perfil hematológico de recém-nascidos de uma Unidade de Terapia Intensiva neonatal em Teresina - PI. Metodologia: Foram analisados os hemogramas contidos em prontuários eletrônicos ou histórico médico hospitalar dos neonatos internados na Unidade de Terapia Intensiva Neonatal. Os recémnascidos foram classificados segundo o sexo e idade em dias e os dados hematológicos foram coletados através do contador hematológico Sysmex® Série-XS 800i. Foram analisadas a série vermelha (eritograma), série branca (leucograma) e plaquetas (plaquetograma). Resultados: A análise dos hemogramas dos recémnascidos demonstrou prevalência de neonatos do sexo feminino (93,3\%) com idade entre $0-3$ dias $(50 \%)$ quando submetidos a realização do hemograma. No eritograma, leucograma e plaquetograma verificou-se uma tendência a normalidade dos valores encontrados, mostrando alterações nos índices hematimétricos VCM (62\%), HCM (68,6\%) e RDW(92\%), além da prevalência de linfopenia (57,3\%). Conclusão: $O$ hemograma do recém-nascido oferece inúmeras informações e sua interpretação pode variar de acordo com a idade e demais características inerentes ao neonato. Entretanto, os valores de referência para essa faixa etária são de difícil obtenção.

Palavras-chave: Recém-nascidos, Hemograma, Unidade de Terapia Intensiva.

\begin{abstract}
Objective: To evaluate the hematologic profile of newborns of a neonatal intensive care unit in Teresina - PI. Methodology: We analyzed the hemograms contained in electronic medical records or hospital medical history of neonates admitted to the Neonatal Intensive Care Unit. The newborns were classified according to sex and age in days and hematological data were collected through the hematologic counter Sysmex® SeriesXS 800i. The red series (erythrogram), white series (leukogram) and platelets (plaquetogram) were analyzed.

Results: The analysis of the hemograms of the newborns showed a prevalence of female neonates (93.3\%) aged $0-3$ days $(50 \%)$ when they underwent hemogram. In the erythrogram, leukogram and plaquetogram, there was a tendency to normality of the values found, showing changes in hematimetric indexes $(62 \%), \mathrm{HCM}$ $(68.6 \%)$ and RDW (92\%), and prevalence of lymphopenia (57, 3\%). Conclusion: The hemogram of the newborn offers numerous information and its interpretation may vary according to the age and other characteristics inherent to the neonate. However, the reference values for this age group are difficult to obtain.
\end{abstract}

Keywords: Newborns, Blood count, Intensive care unit.

${ }^{1}$ Centro Universitário Santo Agostinho - UNIFSA. * E-mail: naty sa123@hotmail.com

${ }^{2}$ Universidade do Brasil - UB.

${ }^{3}$ Centro Universitário UNIEURO.

${ }^{4}$ Universidade Estadual da Paraíba - UEPB

${ }^{5}$ Universidade Federal do Piauí - UFPI. 


\section{RESUMEN}

Objetivo: Evaluar el perfil hematológico de recién nacidos de una Unidad de Terapia Intensiva neonatal en Teresina - PI. Metodología: Se analizaron los hemogramas contenidos en prontuarios electrónicos o histórico médico hospitalario de los neonatos internados en la Unidad de Terapia Intensiva Neonatal. Los recién nacidos se clasificaron según el sexo y la edad en días y los datos hematológicos se recogieron a través del contador hematológico Sysmex® Serie-XS 800i. Se analizaron la serie roja (eritograma), serie blanca (leucograma) y plaquetas (plaquetograma). Resultados: El análisis de los hemogramas de los recién nacidos demostró prevalencia de neonatos del sexo femenino (93,3\%) con edad entre 0-3 días $(50 \%)$ cuando se sometió a la realización del hemograma. En el eritograma, leucograma y plaquetograma se verificó una tendencia a la normalidad de los valores encontrados, mostrando alteraciones en los índices hematimétricos VCM (62\%), HCM (68,6\%) y RDW (92\%), además de la prevalencia de linfopenia (57, 3\%). Conclusión: El hemograma del recién nacido ofrece innumerables informaciones y su interpretación puede variar de acuerdo con la edad y demás características inherentes al neonato. Sin embargo, los valores de referencia para este grupo de edad son de difícil obtención.

Palabras clave: Recién nacidos, Recuento sanguíneo, Unidad de terapia intensiva.

\section{INTRODUÇÃO}

O período neonatal, que compreende os primeiros 27 dias pós-parto, é uma fase considerada de vulnerabilidade à saúde infantil devido a riscos biológicos, ambientais, sociais e culturais. Esse período é também responsável por 60 a $70 \%$ dos óbitos infantis nas últimas décadas, ocorrendo principalmente até o 60 dia de vida, sendo o indicador fundamental de qualidade da atenção ao recém-nascido (BRASIL, 2011). De acordo com Cardoso - Demartini (2011) atualmente, cerca de 13 milhões de crianças nascem prematuramente em todo o mundo e o número de partos prematuros tem aumentado nos últimos anos.

A Unidade de Terapia Intensiva Neonatal (UTIN), por ser um local onde se realizam cuidados intensivos ao $\mathrm{RN}$ de alto risco, pode ser considerada de suma importância para sua recuperação. Conhecimentos científicos possibilitaram importantes avanços tecnológicos na área perinatal, proporcionando a sobrevida a seres cada vez mais imaturos e que há até pouco tempo poderiam ser considerados inviáveis (ROSSETTO et al., 2011).

A incidência de infecções hospitalares varia de acordo com as características de cada unidade de tratamento, infraestrutura e recursos humanos, do próprio recém-nascido (RN), idade gestacional e dos métodos de prevenção e diagnósticos disponíveis. As taxas gerais de infecção hospitalar em unidades neonatais de países desenvolvidos variam de 8,4 a $26 \%$. No Brasil, Unidades de Terapia Intensiva Neonatal (UTIN) de nível terciário possuem taxas de infecção entre 18,9 a 57,7\%, havendo referências de causas possíveis das elevadas taxas de infecção, tais como: condições de trabalho, estrutura física da UTIN e número de profissionais de enfermagem por leito (PINHEIRO et al., 2009).

O hemograma compreende a contagem das células do sangue periférico (hemácias, leucócitos e plaquetas) e a contagem diferencial dos cinco tipos leucocitários (eosinófilos, basófilos, neutrófilos, linfócitos e monócitos) além da quantidade dos valores da hemoglobina e do hematócrito (determinação em porcentagem do volume de hemácia em relação ao volume de sangue) e ainda o do cálculo dos índices hematimétricos (CRISTO et al., 2014).

O sangue periférico é o objeto do hemograma, que reúne as medidas em valores absolutos e percentuais e acrescenta a aparência morfológica das três populações celulares, leucócitos, eritrócitos e plaquetas. A partir do nascimento, o recém-nascido sofre importantes modificações do quadro hematológico com a finalidade de se adaptar ao meio extrauterino. Observa-se nas primeiras semanas de vida uma queda acentuada, autolimitada, da taxa de hemoglobina, comum a todos os lactentes (AGUIAR et al., 2015). 
O conhecimento das características de um grupo populacional contribui para a redução dos índices de indicadores de saúde, principalmente o coeficiente de mortalidade infantil. Dessa forma, a presente pesquisa teve por objetivo avaliar o perfil hematológico em recém-nascidos de uma UTI neonatal em Teresina - PI.

\section{METODOLOGIA}

\section{Tipo de pesquisa}

O trabalho é enquadrado como pesquisa de campo com caráter descritivo, retrospectivo, baseado na análise quantitativa de dados secundários para avaliação hematológica dos recém-nascidos admitidos em uma Unidade de Terapia Intensiva Neonatal (UTIN) de Teresina - PI.

\section{Coleta de dados}

A amostra incluiu 150 prontuários de recém-nascidos, na qual foram analisados os hemogramas contidos em prontuários eletrônicos ou histórico médico hospitalar desses pacientes internados na Unidade de Terapia Intensiva Neonatal da maternidade MDER. As crianças foram classificadas segundo o sexo e a idade em dias. Os dados hematológicos foram coletados através do contador hematológico Sysmex® Série-XS 800i, utilizado pelo laboratório de análises clínicas responsáveis pelos hemogramas dos neonatos, o qual possui valores de referência de acordo com a idade do paciente.

\section{Análise dos dados}

Os dados foram transcritos para o programa Microsoft Excel, processados e analisados com auxílio do programa Statistical Package for the Social Sciences ${ }^{\circledR}$ (SPSS). Foram realizadas a avaliação dos parâmetros eritrocitários, o leucograma e a contagem de plaquetas. A média e desvio padrão foram calculados para as variáveis quantitativas e proporção para as variáveis qualitativas.

\section{Aspectos Éticos e legais}

A pesquisa foi submetida e aprovada pelo Comitê de Ética em Pesquisa em Seres Humanos do Centro Universitário Santo Agostinho sobre número do CAAE 76848617.0.0000. 5602 e autorizado pela Comissão de Ética da MDER. Essa pesquisa visa fazer a avaliação do perfil hematológico de recém-nascidos em uma maternidade pública de Teresina - PI. Dessa forma, foram seguidos os preceitos éticos em pesquisa envolvendo seres humanos conforme determina a Resolução no 466/2012 do Conselho Nacional de Saúde.

\section{RESULTADOS}

No período de fevereiro a maio de 2018, foram analisados 150 hemogramas de neonatos admitidos na Unidade de Terapia Intensiva da maternidade MDER em Teresina - PI. Na tabela 1, está apresentado o perfil dos neonatos de acordo com o sexo e a idade do recém-nascido quanto ao dia da realização do hemograma. Os achados demonstraram predominância de recém-nascidos do sexo feminino $(93,3 \%)$ com média de idade de 21,4 variando entre 0-27 dias; nessa variação, 75 (50\%) neonatos tinham entre 0-3 dias de nascimento quando realizaram o exame hematológico.

No processo de verificação do hemograma dos neonatos, foi analisada a série vermelha (eritrograma), série branca (leucograma - leucócitos, neutrófilos e linfócitos) e plaquetas (plaquetograma).

Dessa forma, a utilização dos termos alto, normal e baixo foi empregada a fim de facilitar a visualização e interpretação dos resultados aqui encontrados na tabela 2. Destacam-se, principalmente, a tendência à normalidade na maior parte das medidas observadas.

A análise dos parâmetros eritrocitários permitiu verificar índice de normalidade nos valores das hemácias $(53,3 \%)$, hemoglobina $(41,3 \%)$ e hematócrito $(38 \%)$. Por outro lado, os índices hematimétricos VCM $(62 \%)$, $\operatorname{HCM}(68,6 \%)$ e RDW $(92 \%)$ tiveram resultados acima do valor de normalidade. Destaca-se no leucograma a prevalência de linfopenia $(57,6 \%)$, leucócitos e neutrófilos, por sua vez, mantiveram em sua maioria valores dentro do padrão de referência. O plaquetograma, também mostrou valores dentro da faixa de normalidade $(69,3 \%)$. 
Tabela 1. Classificação dos recém-nascidos admitidos em uma UTI Neonatal em Teresina - PI.

\begin{tabular}{ccccc}
\hline Variáveis & & Frequência & $\%$ & P valor \\
\hline Sexo & Masculino & 10 & $6,7 \%$ & $<0,001$ \\
& Feminino & 140 & $93,3 \%$ & \\
\hline Idade & $0-3$ dias & 75 & $50,0 \%$ & \\
& $4-7$ dias & 26 & $17,3 \%$ & $<0,001$ \\
& $8-11$ dias & 6 & $4,0 \%$ & \\
$12-15$ dias & 10 & $6,7 \%$ & \\
$16-19$ dias & 8 & $5,3 \%$ & \\
& $20-23$ dias & 10 & $6,7 \%$ & \\
& $24-27$ dias & 15 & $10,0 \%$ & \\
\hline Total de recém-nascidos & $\mathbf{1 5 0}$ & $\mathbf{1 0 0} \%$ &
\end{tabular}

Fonte: MDER, 2018.

\section{DISCUSSÃO}

A Unidade de Terapia Intensiva Neonatal (UTIN) é tida como um ambiente hospitalar nas quais diversas técnicas, procedimentos e intervenções profissionais são utilizadas visando reverter distúrbios que colocam em risco pacientes no período neonatal (intervalo compreendido entre o nascimento e o 27-dia de vida completo), nos mais diferenciados graus de complexidade. Ressalta-se que recém-nascidos, são altamente suscetíveis a infecções invasivas, que são as principais causas de mortalidade e morbidade a longo prazo (SANTOS et al., 2012).

Os exames laboratoriais nos recém-nascidos (principalmente os séricos) representam uma ferramenta importante no auxílio do diagnóstico, evoluções e terapias das condições neonatais e expressam impacto positivo na redução da morbimortalidade se corretamente solicitados e interpretados (GRANZOTTO et al., 2012). O hemograma é considerado um instrumento útil na prática neonatal por permitir o aumento do desfecho favorável aos neonatos, e configurar nos dias atuais uma ferramenta indispensável para o manejo destes pacientes, em vista que é um exame de fácil obtenção e possuir característica capazes de demonstrar uma visão global do estado de saúde desses recém-nascidos (SILVA et al., 2009).

O presente estudo analisou o perfil hematológico de recém-nascidos admitidos em uma Unidade de Terapia Intensiva neonatal da MDER em Teresina - PI. Dentre os 150 hemogramas de recém-nascidos analisados, 93,3\% eram do sexo feminino e 50\% (75 neonatos) tinham idade entre 0 a 3 dias de nascidos quando submetidos ao exame laboratorial. Os achados dessa pesquisa tiveram semelhança com os resultados de Aguiar et al. (2015) no que diz respeito à idade (127 -88,8\% estavam no primeiro dia de vida), porém tiveram divergência na predominância quanto ao sexo, na qual os neonatos, em sua maioria, eram do sexo masculino $(53,5 \%)$.

Hayden et al. (2012) explicam que o recém-nascido passa por notáveis modificações hematológicas logo após o nascimento, para se adaptar ao meio extrauterino. Durante as primeiras semanas de vida, por exemplo, os níveis séricos de hemoglobina e hematócrito podem ser comprometidos devido à redistribuição dos fluidos corporais, níveis esses que tendem a ser altos nos primeiros dias e vida e decaírem até que se normalizem após a oitava semana de vida.

A análise dos parâmetros do eritograma permitiu verificar normalidade nos valores das hemácias (53,3\%), concentração de hemoglobina (41,3\%) e hematócrito (38\%). Segundo Gonçalves et al. (2010) a concentração de hemoglobina e o hematócrito estão entre os parâmetros mais solicitados pelos clínicos. No estudo realizado pelos autores, os dois parâmetros citados mostraram-se acima do valor de referência à medida que aumentava a idade gestacional dos neonatos, o que dessa forma, foi divergente aos achados desse estudo. 
Tabela 2. Perfil hematológico dos recém-nascidos admitidos em uma UTI Neonatal em Teresina - PI.

\begin{tabular}{|c|c|c|c|c|}
\hline \multicolumn{2}{|c|}{ Variáveis } & \multirow[t]{2}{*}{ Frequência } & \multirow[t]{2}{*}{$\%$} & \multirow[t]{2}{*}{ p valor } \\
\hline Eritograma & & & & \\
\hline & Alto & 04 & $2,7 \%$ & \\
\hline \multirow[t]{3}{*}{ Hemácias (RBC) } & Normal & 80 & $53,3 \%$ & $<0,001$ \\
\hline & Baixo & 66 & $44 \%$ & \\
\hline & Alto & 40 & $26,7 \%$ & \\
\hline \multirow{3}{*}{ Hemoglobina (HGB) } & Normal & 62 & $41,3 \%$ & $<0,001$ \\
\hline & Baixo & 48 & $32 \%$ & \\
\hline & Alto & 39 & $26 \%$ & \\
\hline \multirow[t]{3}{*}{ Hematócrito (HCT) } & Normal & 57 & $38 \%$ & $<0,001$ \\
\hline & Baixo & 54 & $36 \%$ & \\
\hline & Alto & 93 & $62 \%$ & \\
\hline \multirow[t]{3}{*}{ VCM } & Normal & 56 & $37,3 \%$ & $<0,001$ \\
\hline & Baixo & 1 & $0,7 \%$ & \\
\hline & Alto & 103 & $68,6 \%$ & \\
\hline \multirow[t]{3}{*}{$\mathrm{HCM}$} & Normal & 46 & $30,7 \%$ & $<0,001$ \\
\hline & Baixo & 1 & $0,7 \%$ & \\
\hline & Alto & 17 & $11,3 \%$ & \\
\hline \multirow[t]{3}{*}{$\mathrm{CHCM}$} & Normal & 128 & $85,3 \%$ & $<0,001$ \\
\hline & Baixo & 5 & $3,4 \%$ & \\
\hline & Alto & 138 & $92 \%$ & \\
\hline \multirow[t]{2}{*}{ RDW } & Normal & 12 & $8 \%$ & $<0,001$ \\
\hline & Baixo & -- & $0 \%$ & \\
\hline \multicolumn{5}{|l|}{ Leucograma } \\
\hline \multirow{4}{*}{ Leucócitos } & Alto & 42 & $28 \%$ & \\
\hline & Normal & 106 & $70,7 \%$ & $<0,001$ \\
\hline & Baixo & 2 & $1,3 \%$ & \\
\hline & Alto & 20 & $13,3 \%$ & \\
\hline \multirow[t]{3}{*}{ Neutrófilos } & Normal & 97 & $64,7 \%$ & $<0,001$ \\
\hline & Baixo & 33 & $22 \%$ & \\
\hline & Alto & 3 & $2 \%$ & \\
\hline \multirow[t]{2}{*}{ Linfócitos } & Normal & 61 & $40,7 \%$ & $<0,001$ \\
\hline & Baixo & 86 & $57,3 \%$ & \\
\hline \multicolumn{5}{|l|}{ Plaquetograma } \\
\hline & Alto & 7 & $4,7 \%$ & \\
\hline \multirow[t]{2}{*}{ Plaquetas } & Normal & 104 & $69,3 \%$ & $<0,001$ \\
\hline & Baixo & 39 & $26 \%$ & \\
\hline \multicolumn{2}{|c|}{ Total de recém-nascidos } & 150 & $100 \%$ & \\
\hline
\end{tabular}

Fonte: MDER, 2018.

Segundo Chopard, Magalhães e Bruniera (2010) nos primeiros 2 dias de vida, há um aumento da concentração da hemoglobina, decorrente da hemoconcentração transitória, resultante da baixa oferta hídrica e da redistribuição do volume plasmático, com posterior diminuição progressiva a partir da primeira semana de vida. Durante o período neonatal ocorre também maior destruição dos glóbulos vermelhos, pois a meia vida dos eritrócitos, nesta faixa etária, é menor que setenta dias. A queda na concentração de hemoglobina, em contrapartida, principia-se com a diminuição da massa eritroide circulante em consequência da diminuição da eritropoese devido ao maior aporte de oxigênio (YOUNG et al., 2008). 
Apesar dos valores de hemácias, hemoglobina e hematócrito estarem dentro dos padrões de referência para neonatos, os valores dos índices hematimétricos evidenciou um aumento nos valores de VCM (62\%), HCM $(68,6 \%)$ e RDW (92\%). Dessa forma, houve prevalência nos neonatos de hemácias com aspecto macrocítico (hemácias de tamanho grande) e hipercrômicos (glóbulos vermelhos mais escuros). Deve-se levar em consideração na avaliação destes exames a possibilidade das alterações nos índices hematimétricos estarem associadas à presença de infecções agudas, muito frequente em crianças menores de 2 anos (RAMOS; CUMAN, 2009), uma vez que o diagnóstico de algum tipo de anemia em neonatos leva em consideração as alterações do eritograma em geral, principalmente quando associados a alterações nos valores de hemoglobina, o que não foi verificado no estudo.

Confirmando o exposto anteriormente, Menezes, Leal e Osório (2010) explicam que a anemia apresenta um alto percentual quando diagnosticada pela hemoglobina como único indicador. Ao se utilizar a associação da hemoglobina com os índices hematimétricos, aumenta-se a especificidade para o diagnóstico etiológico da anemia, evidenciando-se conjuntamente as características morfológicas e, consequentemente, diminuindo o número de indivíduos considerados anêmicos.

No leucograma, foram observados valores de normalidade no número de leucócitos (70,7\%) e neutrófilos $(64,7 \%)$. De acordo com Aguiar et al., (2015) a contagem total e diferencial de leucócitos no sangue apesar de ser um exame bastante sensível, também pode ser considerado inespecífico, uma vez que qualquer condição ou processo inflamatório que acarrete em liberação de citocinas tende a causar uma leucocitose (aumento do número de leucócitos) ou uma leucopenia (diminuição do número de leucócitos). Os recémnascidos em contrapartida apresentaram prevalência de linfopenia $(57,3 \%)$ - diminuição do número de linfócito.

Valores normais de plaquetas foi o achado mais encontrado no plaquetograma dos neonatos. Segundo Uehara et al. (2010) pelo menos 50\% dos bebês internados em UTIN desenvolve trombocitopenia, podendo aumentar em cerca de 75 a $90 \%$ se o caso estiver relacionado com prematuridade, tornando assim, o achado previsível e de natureza benigna. De maneira semelhante, Gonçalves et al. (2010) relata que no estudo da contagem das plaquetas (hemograma) a maior evidência em paciente neonatal é para a trombocitopenia, podendo suas causas ser numerosas e variadas. Como discutido anteriormente, os resultados aqui encontrados não tiveram semelhanças com os descritos na literatura.

\section{CONCLUSÃO}

O perfil hematológico do recém-nascido representa uma importante ferramenta ao neonatologista, uma vez que acompanha e revertem distúrbios que acometem o recém-nascido. Verificou-se na pesquisa uma tendência a normalidade dos valores encontrados para o eritograma, leucograma e plaquetograma, além de alterações nos índices hematimétricos VCM (62\%), HCM (68,6\%) e RDW(92\%), além da prevalência de linfopenia $(57,3 \%)$. Tal estudo possui como contribuição direcionar, planejar e fortalecer ações assistenciais voltadas à promoção da saúde neonatal e à minimização dos agravos relacionados a saúde dos recémnascidos.

\section{REFERÊNCIAS}

1. AGUIAR CF, BALDESSAR MZ, DAL-BÓ K. Perfil hematológico dos neonatos admitidos em Unidade de Terapia Intensiva neonatal de um hospital no Sul do Brasil. Revista da AMRIGS, v. 59, n.4, 2015.

2. BRASIL. Ministério da Saúde (MS). Secretaria de Atenção à Saúde. Departamento de Ações Programáticas e Estratégicas. Atenção à saúde do recém-nascido: guia para os profissionais de saúde. Brasília: Ministério da Saúde (MS), v.1, 2011.

3. CAPPELLETTI M, DELLA SB, FERRAZZI E et al. Inflammation and preterm birth. J Leukoc Biol., v. 99, n.1, p. 67-78, janeiro, 2016.

4. CARDOSO-DEMARTINI AAC, BAGATIN AC, SILVA RPGVC et al. Crescimento de crianças nascidas prematuras. Arq. Bras. Endocrinol. Metab., v.55, n.8, p.534-540, 2011.. 
5. CARNEIRO-SAMPAIO M, SLHESSARENKO N. Vamos reduzir o volume de sangue colhido para exames laboratoriais? Rev Paul Pediatr. v.32, n.2, p.291-2, 2014.

6. CHOPARD MRT, MAGALHÃES M, BRUNIERA P. Deficiência de ferro no feto e recém-nascido. Rev. Bras. Hemat. Hemoter., v.32, s.2, p.32-37, 2010.

7. CRISTO PHC, PEREIRA FS, VALENTIM LS et al. Avaliação do perfil hematológico da linhagem vermelha em idosos atendidos no laboratório de análises clínicas (LAC) da Faculdade de Ciências Farmacêuticas da Universidade Federal do Pará. Anais do III Congresso de Educação em Saúde da Amazônia (COESA), Universidade Federal do Pará, 2014.

8. GONÇALVES J, SOUZA NMA, HERMES EM et al. Perfil hematológico dos neonatos atendidos no Hospital Universitário da Universidade Federal de Santa Catarina. Rev. Bras. Hematol. Hemoter., v.32, n.3, p.219-224, 2010.

9. GRANZOTTO JA, FONSECA SS, LINDEMANN FL. Fatores relacionados com a mortalidade neonatal em uma Unidade de Terapia Intensiva neonatal na região Sul do Brasil. Revista da AMRIGS, Porto Alegre, v. 56, n.1, p.57-62, 2012.

10. HAYDEN SJ, ALBERT TJ, WATKINS TR et al. Anemia in Critical Illness Insights into Etiology, Consequences, and Management. American Journal of Respiratory and Critical Care Medicine, ed.185, 2012.

11. HUNTER PJ, SHEIKH S, DAVID AL et al. Cervical leukocytes and spontaneous preterm birth. Journal of Reproductive Immunology, v.113, p.42-49, 2016.

12. KARENBERG K, HUDALLA H, FROMMHOLD D. Leukocyte recruitment in preterm and term infants. Molecular and Cellular Pediatrics, v.3, n.35, 2016.

13. MENEZES AEB, LEAL LP, OSÓRIO MM. Validação de índices hematimétricos para o diagnóstico etiológico da anemia ferropriva em crianças de 6 a 23 meses. Rev Enferm UFPE, v.4, n.1, p.749-56, 2010.

14. MENEZES-BRITO MJ, MATTIA RA, RESENDE FJ. Análise dos eventos adversos em uma unidade de terapia intensiva neonatal como ferramenta de gestão da qualidade da assistência de enfermagem. Enferm. glob. Murcia, n.17, 2009.

15. PINHEIRO MSB, NICOLETTI C, BOSZCZOWSK I et al. Infecção hospitalar em Unidade de Terapia Intensiva Neonatal: há influência do local de nascimento? Rev. Paul. Pediatr., v.27, n.1, p.6-14, 2009.

16. RAMOS HAC, CUMAN RKN. Fatores de Risco Para Prematuridade: Pesquisa Documental. Esc. Anna Nery, vol.13, n.2, p.297-304, 2009.

17. ROSSETTO M, PINTO EC, SILVA LAA. Cuidados ao recém-nascido em terapia intensiva: tendências das publicações na enfermagem. Rev. de Ciências da Saúde, v.23, n.1, 2011.

18. SANTOS LM, SILVA TPCC, SANTANA RCB et al. Sinais sugestivos de dor durante a punção venosa periférica em prematuros. Rev Enferm UFSM. v.2, n.1, p.01-09, 2012.

19. SAVA F, TOLDI G, TRESZL A et al. Expression of lymphocyte activation markers of preterm neonates is associated with perinatal complications. Bio Med Central Immunol, v.17, n.19, 2016.

20. SILVA LJ, SILVA LR, CHRISTOFFEL MM. Tecnologia e humanização na Unidade de Terapia Intensiva Neonatal: reflexões no contexto do processo saúde-doença. Rev. Esc. Enferm. USP, São Paulo, v.43, n.3, 2009.

21. TADIELO BZ, NEVES ET, ARRUÉ AM et al. Morbidade e mortalidade de recém-nascidos em tratamento intensivo neonatal no sul do Brasil. Revista da Sociedade Brasiliera de Enfermeiros Pediatras. v.13. n.1, 2013.

22. UEHARA KM, CARDOSO LTQ, CARRILHO CMDM et al. Trombocitopenia como fator prognóstico em pacientes com sepse grave internados em Unidade de Terapia Intensiva. Semina: Ciências Biológicas e da Saúde, Londrina. V.31, N2, p195-200, 2010.

23. YOUNG TE, MANGUM B, REUTERS T. Neofax. 21st ed. Montvale (NJ): Wiley-Blackwell; p. 296-313, 2008. 